\title{
VOC Sensing Properties of Hybrid Nanostructures
}

\author{
E. Şennik ${ }^{1,5}$, B.Şennik ${ }^{2,3}$, O. Alev ${ }^{1}$, N. Kılınç ${ }^{4,5}$, F. Yılmaz ${ }^{2}$, Z.Z. Öztürk $^{1}$ \\ ${ }^{1}$ Department of Physics, Gebze Technical University, Kocaeli 41400, Turkey \\ ${ }^{2}$ Department of Chemistry, Gebze Technical University, Kocaeli 41400, Turkey \\ ${ }^{3}$ Department of Chemistry, Istanbul Medeniyet University, 34720 Istanbul, Turkey \\ ${ }^{4}$ Mechatronics Engineering Department, Nigde University, Nigde 51245, Turkey \\ ${ }^{5}$ Nanotechnology Application and Research Center, Nigde University, Nigde 51245, Turkey \\ zozturk@gtu.edu.tr
}

\begin{abstract}
In this study, polymer/ $/ \mathrm{TiO}_{2}$ nanoheterostructures were fabricated to investigate their gas sensing properties. $\mathrm{TiO}_{2}$ nanorods were fabricated by hydrothermal method. Then polystyrene polymer was coated on $\mathrm{TiO}_{2}$ nanorods by spin coating method. The morphologies and structure of the samples were characterized by X-ray diffraction (XRD), scanning electron microscope (SEM). Electrical and VOC sensing properties of hybrid nanostructure device were investigated. The hybrid nanostructure shows highest sensor response for ethanol $(\mathrm{EtOH})$ at $100{ }^{\circ} \mathrm{C}$.
\end{abstract}

Key words: $\mathrm{TiO}_{2}$ nanorods, polymer, heterostructure, VOC, gas sensor.

\section{Introduction}

The gas sensors are the subject of an intensive research because of their applications in the industry (such as petro chemistry, mining, cosmetic, automobile, and food industry), healthcare, agriculture, environmental protection, military, and security [1, 2]. The several materials such as the metals and metal oxides are widely used as the sensing layer which can be successfully produced as thin films, nanowires [3], nanotubes [4], in order to improve the properties of the sensor. Also, organic materials such as phthalocyanines [5] and polymers can be coated in order to develop sensor parameters.

In this work, polymer/ $/ \mathrm{TiO}_{2}$ nanoheterostructures were fabricated to investigate their gas sensing properties.

\section{Experimental}

$\mathrm{TiO}_{2}$ nanorods were hydrothermally fabricated onto fluorine-doped tin oxide (FTO) substrate. 1 $\mathrm{ml}$ Titanium (IV) $\mathrm{n}$ - butoxide was used as a Ti precursor and mixed by $\mathrm{HCl}$ : deionized water with equal volume. The hydrothermal reaction was performed in teflon lined stainless steel autoclave at $150{ }^{\circ} \mathrm{C}$ for $18 \mathrm{~h}$. Fig. 1a shows schematic illustration about teflon and stainless steel autoclave which used for growth nanorods on FTO by hydrothermal method. SEM image of $\mathrm{TiO}_{2}$ nanorods is given by Fig. $1 \mathrm{~b}$.

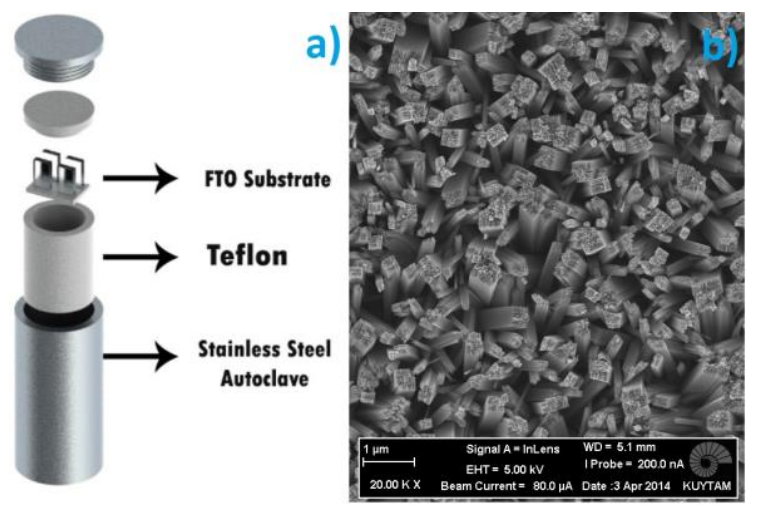

Fig. 1. a) A schematic illustration about hydrothermal method, b) SEM image of $\mathrm{TiO}_{2}$ nanorods.

$\mathrm{P}\left(\mathrm{S}-\mathrm{co}-\mathrm{CMS}^{-\mathrm{C}_{60}}\right)(\mathrm{P} 3)$ was prepared as follows: A mixture of $\mathrm{P}$ (S-co-CMS-N3) (P2), $\mathrm{C}_{60}$ and chlorobenzene was placed in a round bottom flask and degassed by bubbling argon for 10 min. The homogeneous reaction mixture was stirred at $60{ }^{\circ} \mathrm{C}$ under argon for $2 \mathrm{~d}$. The solution was heated $130{ }^{\circ} \mathrm{C}$ and allowed to continue overnight again. The resulting mixture was evaporated to dryness and then THF was added the residue. The mixture was stirred at room temperature. Unreacted $\mathrm{C}_{60}$ and other insoluble matters were filtered off. The clean filtrate was evaporated and dried in vacuum desiccator to obtain brown product. Then synthesized polystyrene polymer was coated on $\mathrm{TiO}_{2}$ nanorods by spin coating method. 


\section{Results and Discussion}

$\mathrm{TiO}_{2}$ nanorods were fabricated by hydrothermal method as seen in Fig. 1b. The length and diameter of the fabricated $\mathrm{TiO}_{2}$ nanorods were about $1 \mu \mathrm{m}$ and $100 \mathrm{~nm}$ respectively. According to the XRD results, the peaks represent the reflections from rutile $\mathrm{TiO}_{2}$ crystallites, and FTO substrate as seen in Fig. 2.

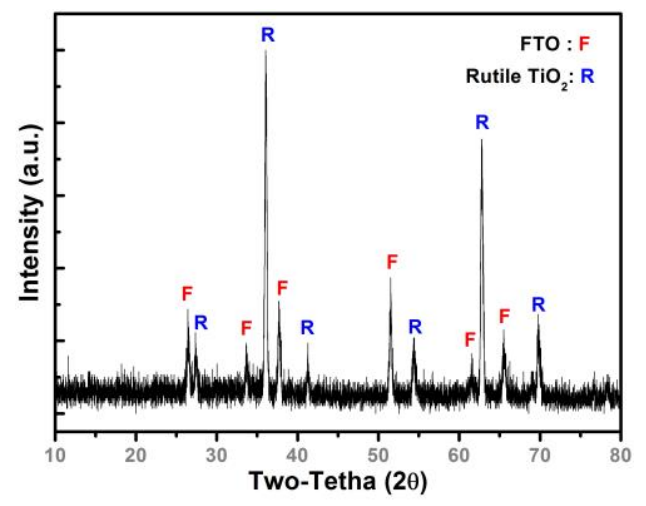

Fig. 2. XRD of $\mathrm{TiO}_{2}$ nanorods.

Polystyrene polymer was coated on $\mathrm{TiO}_{2}$ nanorods by spin coating method as a thin film.

For gas sensing measurement, gold contacts were coated on hybrid nanostructures. Fig. 3a shows schematically illustration of polymer/TiO nanorods hybrid nanostrucutres. The DC electrical properties of hybrid device were investigated under dry air flow depending on temperature. The I-V characteristics of hybrid device have a hysteresis at all temperatures and the hysteresis increased with increasing temperature as seen in Fig. $3 b$.
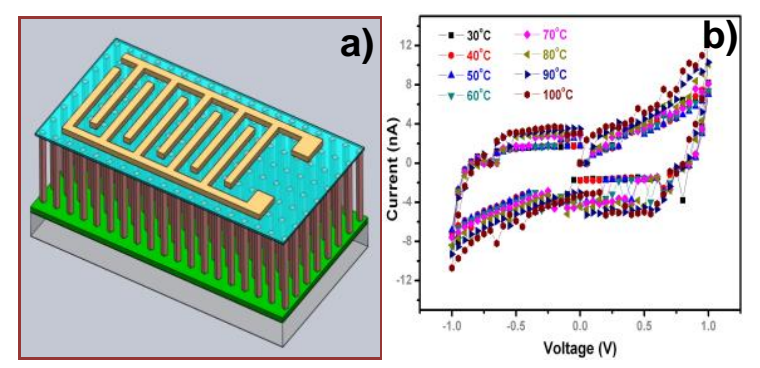

Fig. 3. a) A schematic illustration about hybrid device, b) IV characteristics of hybrid device.

VOC sensing properties of hybrid nanostructure device were investigated at $100{ }^{\circ} \mathrm{C}$ and the sensor response of the device is given in Fig 4. VOCs were sent to test cell for $10 \mathrm{~min}$, and then the cell recovered by dry air for $20 \mathrm{~min}$. The hybrid nanostructure shows highest sensor response for ethanol (EtOH).

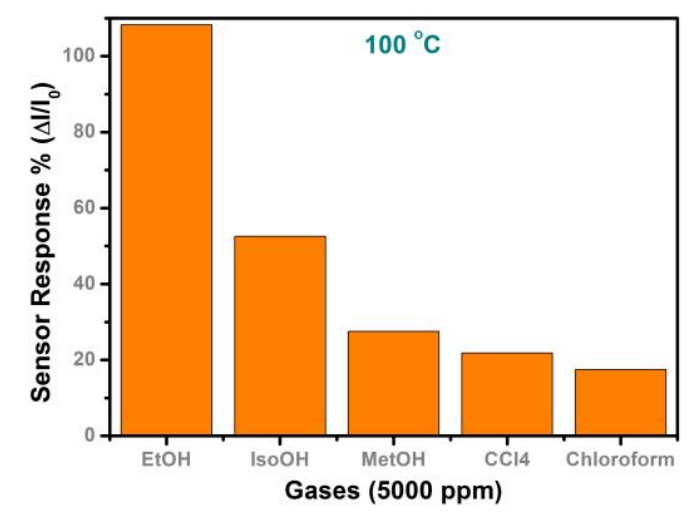

Fig. 4. VOC sensing properties of hybrid device at $100^{\circ} \mathrm{C}$.

It is well known that mostly carbon atom is accessible to the gas because they are the surface atoms and gas sensing for P3 may depend on the surface interaction between the gas and $\pi$ electrons in $\mathrm{C}_{60} \mathrm{~s}$. While the copolymer chain provides solubility, $\mathrm{C}_{60}$ moieties ensure the sensing [6].

\section{Acknowledgement:}

This study was supported by The Scientific and Technological Research Council of Turkey (TUBITAK) with project number of $113 \mathrm{~F} 403$.

\section{References}

[1] E. Comini, G. Faglia, G. Sberveglieri, Solid State Gas Sensing, New York: Springer, 2009.

[2] W. Gopel, et al., Sensors: A Comprehensive Survey (volume 2-3), Chemical and Biochemical Sensors, New York: WCH, 1992.

[3] E. Sennik, U. Soysal, Z. Z. Öztürk, Pd loaded spider-web $\mathrm{TiO}_{2}$ nanowires:Fabrication, characterization and gas sensing properties, Sensors and Actuators B. 199, 424 (2014).

[4] E. Şennik, Z. Çolak, N. Kılınç, Z. Z. Öztürk, Synthesis of highly-ordered $\mathrm{TiO}_{2}$ nanotubes for a hydrogen sensor, International Journal of Hydrogen Energy 35, 4420 (2010).

[5] A. Belghachi and R. A. Collins, Humidity response of phthalocyanine gas sensors, Journal of Physics D: Applied Physics 21, 1647 (1988).

[6] S. Sharma, S. Hussain, S. Singh, S.S. Islam, MWCNT-conducting polymer composite based ammonia gas sensors: A new approach for complete recovery process, Sensors and Actuators B. 194, 213(2014). 